\title{
Experiencia interdisciplinar en humanidades: propuesta de intercomunicación entre la mitología clásica y la enseñanza del inglés Interdisciplinary practice in humanities: a proposal for the intercommunication of classical mythology and English language teaching
}

\author{
María de la Luz García Fleitas ${ }^{1}$, Carolina Rodríguez Juárez ${ }^{2}$ \\ mariadelaluz.garcia@ulpgc.es, carolina.rodriguez@ulpgc.es \\ ${ }^{1}$ Departamento de Filología Hispánica Clásica y de $\quad{ }^{2}$ Departamento de Filología Moderna, Traducción \\ Estudios Árabes y Orientales \\ e Interpretación \\ Universidad de Las Palmas de Gran Canaria \\ Las Palmas de Gran Canaria, España
}

\begin{abstract}
Resumen- Las materias que conforman un título no deberían concebirse aisladas unas de otras, ni en contenidos ni en metodologías. En el presente trabajo se describe una práctica de innovación educativa cuyo objetivo ha sido desarrollar una experiencia interdisciplinar en la que el alumnado pudo aplicar e interrelacionar los conocimientos adquiridos en tres asignaturas: Inglés I, Mitología Clásica y Cultura Europea y Estrategias para el aprendizaje de las lenguas modernas impartidas en primer curso del Grado en Lenguas Modernas (curso 2020-2021) en la Universidad de Las Palmas de Gran Canaria. La evaluación del trabajo llevado acabo por el alumnado fue positiva, pudiéndose contrastar que los conocimientos presentados en las tres asignaturas se adquirieron y activaron de forma adecuada. Esta percepción positiva del profesorado fue corroborada por la opinión de los estudiantes, que valoraron positivamente esta experiencia interdisciplinar.
\end{abstract}

Palabras clave: Coordinación interdisciplinar, EEES, Humanidades

Abstract- The subjects that are contemplated in a degree study plan should not be conceived in isolation, neither in terms of their contents nor in their methodologies. In this study we describe a practical experience of educational innovation whose aim has been to develop an interdisciplinary approach in which students have been able to apply and interrelate the knowledge acquired in three subjects: English I, Classical Mythology and European Culture, and Strategies for the Learning of Modern Languages in the Degree in Modern Languages (2020-2021) at the University of Las Palmas de Gran Canaria. The assessment of the tasks carried out by the students showed encouraging results and it could be proved that the contents presented in the three subjects were adequately acquired and activated. The teachers' positive perceptions were also supported by the students' opinions, who evaluated this interdisciplinary practice in a positive way.

Keywords: Interdisciplinary coordination, EHEA, Humanities

1. INTRODUCCIÓN ${ }^{1}$

${ }^{1}$ Este trabajo ha sido realizado en el marco del proyecto de investigación «Marginalia Classica: Recepción Clásica y
Las asignaturas que conforman un título no pueden concebirse aisladas unas de otras, ni en contenidos ni en metodologías. En este sentido, y ante los retos que plantea el EEES, la coordinación docente constituye uno de los pilares fundamentales para el desarrollo de las competencias profesionales que debe adquirir el alumnado. Las asignaturas tienden a agruparse en materias y en módulos con el objetivo de salvaguardar la continuidad y la relación disciplinar y la coherencia en el aprendizaje, pero ello no siempre supone garantía de una relación significativa. Recogiendo las conclusiones básicas del Libro Blanco de la Investigación en Humanidades en lo que se refiere a su función social, la oferta académica del Grado en Lenguas Modernas de la Universidad de Las Palmas de Gran Canaria (ULPGC), donde hemos centrado nuestra experiencia, incluye no solo aspectos lingüísticos competenciales en español, inglés, francés (o chino) y un cuarto idioma, sino también competencias literarias, audiovisuales, históricas, culturales e interculturales; de ahí su configuración en torno a seis módulos que atienden a esa variedad de competencias (Módulo I. La Lengua inglesa: aspectos teóricos y prácticos; Módulo II. Segunda lengua extranjera; Módulo III. Otras lenguas; Módulo IV. Literatura; Módulo V. Estudios Culturales; Módulo VI. Asignaturas instrumentales, metodológicas, de orientación al desempeño profesional y de formación en valores); y que se desarrollan en una serie de materias, que comprenden, a su vez, un número variable de asignaturas (obligatorias y optativas).

En estos momentos la Facultad de Filología de la ULPGC presenta para sus grados un sistema de coordinación tanto horizontal (coordinaciones de semestre y curso) como vertical (coordinación de materias y módulos). Si bien esta labor se lleva a cabo de forma satisfactoria, consideramos que, en virtud de una de las competencias generales del grado (Capacidad para relacionar los conocimientos específicos con los de otras áreas

cultura de masas contemporánea. La construcción de identidades y alteridades» (código PID2019-107253GB-I00). 
y disciplinas del conocimiento), podría enriquecerse aquella con un enfoque interdisciplinar, que supondría la adquisición de un conocimiento más integral que el que se obtiene con el estudio de una asignatura específica, alcanzando de este modo un tipo de aprendizaje más acorde con el que demanda la sociedad actual, caracterizada precisamente por la interdisciplinariedad.

\section{CONTEXTO}

La interdisciplinaridad hace referencia a la interacción entre dos o más disciplinas entre las que debe existir intercomunicación y enriquecimiento recíproco. Estudios sobre enseñanza universitaria señalan la escasa transferencia existente en los conocimientos tratados en las asignaturas cuando estas se consideran de manera individual y destacan el potencial de aquellas experiencias que suponen la conexión de contenidos (Cuadrado, Ruiz Molina y Coca, 2009; Lin, 2008; Wall y Shankar, 2008). Nuestra experiencia de innovación educativa se ha centrado en la vinculación de tres asignaturas de módulos distintos del Grado en Lenguas Modernas de la ULPGC, Estrategias para el aprendizaje de las lenguas modernas (EALM), Inglés I y Mitología Clásica y Cultura Europea (MCCE) - todas ellas obligatorias del primer semestre de primer curso - buscando esa intercomunicación y enriquecimiento recíproco en aras de una mejora en el proceso de aprendizaje.

Por un lado, este grado otorga una importancia primordial a la adquisición de competencias en las lenguas objeto de su aprendizaje, siendo concebida la lengua inglesa como lengua maior, de ahí que se haya elegido para esta práctica de innovación la asignatura Inglés I (del Módulo I La Lengua Inglesa: aspectos teóricos y prácticos; Materia: Idioma moderno), orientada sobre todo a una aprehensión práctica de la lengua inglesa, que asegure la comprensión y el uso eficiente de esta (consecución de un nivel de dominio oral y escrito equivalente al B1 del Marco común europeo de referencia para las lenguas).

En segundo lugar, y en tanto que en la génesis de este grado se considera que el dominio de una lengua no debe consistir solo en una aprehensión estrictamente práctica e instrumental, sino que el estudio de la lengua debe incardinarse en su contexto histórico, social y cultural, hemos dirigido nuestro interés igualmente hacia una asignatura de índole no lingüística: MCCE (del Módulo IV: Literatura; Materia: Literatura), centrada en el estudio del papel que desempeña la mitología clásica en la configuración del imaginario occidental. Además, la mitología no debe estudiarse de forma unidisciplinar, lo que justifica asimismo su inclusión en la presente experiencia docente. Los mitos clásicos, como rico universo de ficción, se hallan presentes a lo largo de nuestra dilatada historia cultural como recreaciones explícitas o como otras formas de apropiación más sutiles, que pueden ser tanto conscientes o inconscientes, y que perviven tanto en la alta cultura como en formatos de lo que podemos denominar cultura de masas. El cine, por ejemplo, constituye un magnífico receptor del mito, al que otorga modernidad (Balló y Pérez, 2010, pp.10-12), haciéndolo inteligible a nuestra sociedad actual. Por ello se ha seleccionado un film, que en nuestro caso serviría de punto de partida para la interrelación de las tres asignaturas: Lars and the Real Girl (2007), escrita por Nancy Oliver y dirigida por Craig Gillespie. Y si bien el aprendizaje de la lengua inglesa a través del cine constituye una práctica ya afianzada (Brown, 2010; Goctu, 2017; Ismaili, 2013; Seferoglu, 2008), el uso de este producto audiovisual (en lengua inglesa) se justifica aquí aún más por el perfil del alumnado al que va dirigida esta práctica, pues, además de interesado por el estudio de otras lenguas y culturas y aspectos tan relevantes en nuestra sociedad como la diversidad y la multiculturalidad, se halla especialmente familiarizado con productos culturales masivos.

En tercer lugar, se eligió Estrategias para el aprendizaje de las lenguas modernas (del Módulo VI: Asignaturas instrumentales, metodológicas, de orientación al desempeño profesional y de formación en valores; Materia: Lingüística), una asignatura de tipo instrumental, que en tanto que ofrece al estudiante estrategias para el aprendizaje de las lenguas modernas y herramientas necesarias para abordar con éxito el estudio de las asignaturas del grado impartidas en inglés o en otras lenguas modernas, ha servido para afianzar aún más la intercomunicación a través, en su caso, de recursos formales para la redacción del trabajo de investigación (MCCE) y la elaboración de presentaciones orales (Inglés I, EALM).

Así pues, la práctica de innovación educativa que presentamos ha tenido como objetivo desarrollar una experiencia interdisciplinar que, aunando asignaturas pertenecientes, como se ha apuntado, a módulos diferentes, favoreciera un desarrollo más globalizado de las competencias, motivando así más al alumnado con un tipo de aprendizaje más acorde con el que demanda la sociedad actual.

\section{DESCRIPCIÓN}

La actividad presentada se llevó a cabo en el contexto del primer semestre del primer curso del Grado en Lenguas Modernas (Facultad de Filología) de la ULPGC durante el curso académico 2020-2021, interviniendo en total tres asignaturas: Estrategias para el aprendizaje de las lenguas modernas (EALM), Inglés I y Mitología Clásica y Cultura Europea (MCCE). El equipo docente interdisciplinar que implementó la actividad estaba constituido por 7 docentes de dos departamentos (Filología Hispánica Clásica y de Estudios Árabes y Orientales, y Filología Moderna, Traducción e Interpretación) y de dos áreas de conocimiento (Filología Griega y Filología Inglesa). La planificación del proyecto propuesta por el equipo se desarrolló a lo largo de la segunda parte del primer semestre en distintas fases: (1) Constitución del equipo docente y delimitación del objeto de estudio: selección de contenidos de las distintas asignaturas, diseño de las pruebas de evaluación, elección de los instrumentos de investigación (cuestionarios, rúbricas); (2) Elaboración de material didáctico (dossiers, pautas, presentaciones en PowerPoint), de rúbricas de evaluación y de formularios para medir la percepción con el desarrollo de la actividad interdisciplinar; (3) Presentación de los contenidos en las distintas asignaturas; (4) Valoración de las pruebas de evaluación y recogida de datos sobre el nivel de satisfacción del alumnado con la actividad; (5) Tratamiento de los datos de satisfacción y análisis de los resultados obtenidos.

En las tablas 1, 2 y 3 presentamos de manera abreviada el cronograma de trabajo, los contenidos impartidos vinculados directamente con la experiencia interdisciplinar, la metodología y las pruebas de evaluación utilizadas en cada una de las tres asignaturas que forman parte de esta actividad:

Tabla 1

Mitología clásica y cultura europea

\begin{tabular}{l|l}
\hline SEMANA & CONTENIDOS Y METODOLOGÍA
\end{tabular}


Tema 3. Temas y estereotipos míticos. EI mito de Pigmalión.

Lección magistral participativa: mito de Pigmalión y su pervivencia en la cultura occidental. Breve recorrido: literatura y cine fundamentalmente. Lectura y comentario del relato de Ovidio (Metamorfosis X 243-297), a 6-7 través del cual se ha conocido mayormente el 6-7 mito. Práctica en el aula: presencia del mito en $(2 \mathrm{~h} .+2 \mathrm{~h}$. un texto literario en lengua inglesa.

$+2 \mathrm{~h}$.)

Visionado de la película Lars and the Real Girl (2007): debido a las circunstancias derivadas de la pandemia COVID-19 se establece como actividad externa.

Debate dirigido en el aula a partir del visionado del film; y presentación de las pautas para la elaboración del trabajo de investigación individual.

\begin{tabular}{c|l}
\hline SEMANA & \multicolumn{1}{|c}{ EVALUACIÓN } \\
\hline \multirow{4}{*10}{} & $\begin{array}{l}\text { Entrega de un trabajo de investigación tutelado } \\
\text { a través del cual se evalúa el Tema 3 y se ponen }\end{array}$ \\
en práctica los conocimientos sobre citación, \\
paráfrasis y elaboración de referencias \\
bibliográficas aprendidos en la asignatura \\
EALM.
\end{tabular}

Tabla 2

Estrategias para el aprendizaje de las lenguas modernas

\begin{tabular}{c|l}
\hline SEMANA & \multicolumn{1}{|c}{ CONTENIDOS Y } \\
\hline 7 & $\begin{array}{l}\text { METODOLOGÍA } \\
\text { Tema Finding information and } \\
\text { referencing sources } \\
\text { Pautas sobre citación, paráfrasis y elaboración } \\
\text { de referencias bibliográficas. }\end{array}$ \\
\hline $\mathbf{1 1}+2 \mathrm{~h})$. & $\begin{array}{l}\text { Tema 5. Speaking and listening strategies } \\
\text { Descripción de estrategias orientadas al a } \\
\text { desarrollo de la competencia comunicativa e } \\
\text { introducción de los componentes que } \\
\text { intervienen en la elaboración de presentaciones } \\
\text { orales. } \\
\text { Presentación de la tarea de elaboración en } \\
\text { grupos de las presentaciones orales sobre la } \\
\text { película Lars and the Real Girl. }\end{array}$ \\
\hline 13-14 & $\begin{array}{l}\text { Exposición en el aula de los trabajos orales en } \\
\text { lengua inglesa sobre los contenidos aprendidos } \\
\text { en la asignatura MCCE a través de la activación } \\
\text { del vocabulario aprendido en los temas 1, 2 y 3 } \\
\text { de la asignatura Inglés I. }\end{array}$ \\
\hline
\end{tabular}

Tabla 3

Inglés $I$

\begin{tabular}{c|l}
\hline SEMANA & \multicolumn{1}{|c}{ CONTENIDOS Y METODOLOGÍA } \\
\hline $6-9$ & $\begin{array}{l}\text { Tema 1: Family terms and relationships } \\
\text { Tema 2: Feelings }\end{array}$ \\
\hline
\end{tabular}

\begin{tabular}{c|l}
\hline $\begin{array}{c}\text { (2h.+2h.+ } \\
2 \mathrm{~h} .+2 \mathrm{~h} .)\end{array}$ & $\begin{array}{l}\text { Tema 3: Describing character } \\
\text { Introducción de vocabulario perteneciente a } \\
\text { distintos campos léxicos a través de tareas } \\
\text { específicas de presentación, consolidación y } \\
\text { activación del nuevo vocabulario. } \\
\text { Introducción y práctica de los tiempos verbales } \\
\text { usados en textos narrativos (tema 3). }\end{array}$ \\
\hline $\mathbf{1 0 - 1 2}$ & $\begin{array}{l}\text { Presentación de la tarea para la elaboración de } \\
\text { la presentación oral sobre la película Lars and } \\
\text { the Real Girl. }\end{array}$ \\
\hline SEMANA & \multicolumn{1}{c}{ EVALUACIÓN } \\
\hline $\mathbf{1 4 - 1 5}$ & $\begin{array}{l}\text { Exposición de los trabajos orales en grupo en } \\
\text { el aula sobre los contenidos aprendidos en la } \\
\text { asignatura MCCE y teniendo en cuenta las } \\
\text { pautas para la elaboración de presentaciones } \\
\text { orales aprendidas en la asignatura EALM. }\end{array}$ \\
\hline
\end{tabular}

Como se puede observar, la coordinación interdisciplinar, de la que se informa al alumnado desde un principio, consistió en integrar (i) conocimientos teóricos sobre mitología clásica (en MCCE), (ii) con contenidos más prácticos e instrumentales sobre formas de citar, parafrasear y elaborar un listado de bibliografía (aspectos que pondrán en práctica en la elaboración del trabajo de investigación de la asignatura MCCE) así como sobre pautas para la elaboración de presentaciones orales (en EALM), y (iii) con conocimientos lingüísticos en lengua inglesa sobre campos léxicos relevantes para la descripción las relaciones personales y de los personajes de la película Lars and the Real Girl, que vieron y trabajaron en MCCE (en Inglés I).

Tras una primera etapa de presentación de los contenidos en las tres asignaturas, el alumnado pudo consolidar y activar los aspectos teóricos trabajados a través de actividades de clase y la elaboración del trabajo de investigación individual centrado en el análisis de la recepción del mito clásico de Pigmalión en la película Lars and the Real Girl, organizado en preguntas precisas sobre la recepción del mito y las posibilidades de lectura del film y del mito en el film; de diversas actividades de contenido léxico en Inglés I tanto dirigidas (actividades del libro de texto) como más libres y personalizadas (speaking activities); y de la preparación de las presentaciones orales en las que pudieron aunar los contenidos teóricos y prácticos aprendidos en las tres asignaturas y desarrollar el aprendizaje colaborativo fuera del aula así como el uso de nuevas tecnologías para la preparación del formato de la presentación oral.

Hemos de añadir que el equipo docente de la actividad interdisciplinar acordó la preparación de una misma presentación oral (en cuanto a la formación de los grupos de estudiantes y al tema elegido) para las asignaturas de Inglés I y de EALM. Sin embargo, esta exposición, llevada a cabo en grupo de 4-5 estudiantes durante 5-7 minutos, sería evaluada en cada asignatura según criterios diferentes: en EALM se evaluarían principalmente aspectos relacionados con la estructura, aspectos visuales y ejecución de la presentación (voz, lenguaje corporal, etc.), y en Inglés I la evaluación se centraría en aspectos relativos al nivel de dominio de la lengua inglesa (vocabulario, corrección gramatical, fluidez, etc.). Para ello, se elaboraron rúbricas detalladas de evaluación por 
asignatura que se publicaron en la plataforma virtual de las asignaturas (Moodle) con anterioridad a la presentación oral.

Cada presentación oral estuvo seguida por unos minutos de discusión entre el alumnado que presentaba y el resto de la clase; se formularon preguntas y se ofreció feedback resaltando aspectos positivos y posibles carencias o acciones de mejora en ambas asignaturas. Esta tarea de reflexión sobre el producto final permitió al alumnado activar las estrategias indirectas metacognitivas (Oxford, 1999) a través del análisis de su progreso y de su aprendizaje con el fin de que la adquisición de conocimientos y el desarrollo de competencias fuesen más significativos. Además, en la asignatura de EALM, el alumnado evaluó de forma individual y anónima la presentación oral de cada grupo a través de un breve formulario online (FormsMicrosoft) ubicado en la plataforma virtual de la asignatura y realizado una vez finalizada la discusión. El cuestionario presentó una escala de valores de 3 puntos (excelente, satisfactorio y flojo) en torno a cuatro aspectos muy concretos de la presentación oral: estructura y organización, relevancia del contenido, uso del lenguaje y apoyo visual. Este feedback fue posteriormente enviado a los miembros del grupo de manera individual junto al feedback y la rúbrica de las docentes de EALM, que incluye tanto la nota grupal como la individual (resultados de la evaluación y posibles acciones de mejora).

Al final del semestre, en la asignatura EALM, se distribuyó un breve cuestionario de satisfacción - a través de un formulario online (Forms-Microsoft) - que sirvió al equipo docente para evaluar la percepción del alumnado en cuanto la relevancia de la actividad interdisciplinar como metodología facilitadora para la activación y asimilación de contenidos de diversas asignaturas de una forma integral.

\section{Resultados}

El impacto de la actividad de innovación educativa desarrollada ha sido medido a través de indicadores de satisfacción para conocer el impacto de la actividad interdisciplinar en la mejora del aprendizaje del alumnado. El instrumento de investigación utilizado fue un formulario de satisfacción diseñado con Forms-Microsoft que se dirigió a toda la población en el mismo momento, por lo que cada individuo tuvo las mismas oportunidades para participar. La población del estudio estaba formada por 51 estudiantes de primero del Grado en Lenguas Modernas (ULPGC), cuya participación en la encuesta, que tiene carácter voluntario, fue elevada (ver tabla 4 ), con un margen de error de 5,98\% para un nivel de confianza del $95 \% \quad(\mathrm{z}=1,96)$ y bajo el supuesto de máxima indeterminación $(\mathrm{P}=\mathrm{Q}=50 \%)$ con una población finita $(\mathrm{N}=$ 51), por lo que los resultados obtenidos en el estudio pueden extrapolarse al conjunto de la población (López-Roldán y Facheli, 2015, p. 24):

\section{Tabla 4}

Datos de la muestra y nivel de participación en la encuesta de satisfacción

\begin{tabular}{cccc}
\hline Población & Muestra & Participación & Error \\
\hline 51 & 43 & 84,31 & 5,98 \\
\hline
\end{tabular}

El cuestionario online de satisfacción estuvo disponible en la plataforma virtual restringido al alumnado presente en el aula durante 10 minutos en la última sesión del curso de la asignatura
EALM, momento tras el que se cerró para poder controlar que solo accedía alumnado que había estado presente en la exposición de las presentaciones orales. El formulario contiene 10 preguntas que se miden a través de una escala Likert de 5 puntos (Totalmente en desacuerdo; Algo en desacuerdo; De acuerdo; Muy de acuerdo; Totalmente de acuerdo) a excepción de la última que es una pregunta abierta sobre sugerencias de mejora. Cuatro preguntas se centran sobre el aspecto interdisciplinar de las actividades llevadas a cabo, tres ítems preguntan sobre la experiencia de trabajar en equipos, y dos ítems indagan sobre aspectos formales tales como la elección del tema y la adecuación de las pautas dadas para la elaboración de presentaciones orales.

Los resultados de satisfacción con respecto a la valoración de la actividad interdisciplinar fueron elevados, con casi un $74 \%$ del alumnado mostrándose muy satisfecho con el impacto de esta actividad en la consolidación y aprendizaje de contenidos:

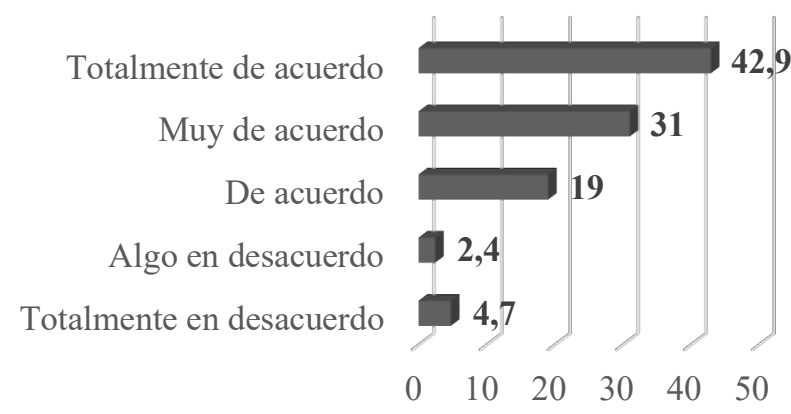

Figura 1: La actividad de preparar una presentación oral en inglés me ha ayudado a consolidar los contenidos aprendidos en las asignaturas de 'Mitología Clásica y Cultura Europea', 'Inglés I' y 'Estrategias para el Aprendizaje de las Lenguas Modernas'.

Si nos centramos en la eficiencia de la actividad (identificar "el esfuerzo para realizar la innovación por primera vez" (Fidalgo Blanco, Sein-Echaluce Lacleta y García-Peñalvo, 2019, p. 627)), también observamos niveles altos de satisfacción (Figura 2: 66,7\% están totalmente o muy satisfechos) que expresan que se han conseguido buenos resultados pero empleando menos esfuerzo, en el sentido de que el visionado de una película en la asignatura de MCCE y el estudio del mito de Pigmalión les ha servido para trabajar el contenido y la activación del léxico requerido en las presentaciones orales en lengua inglesa:

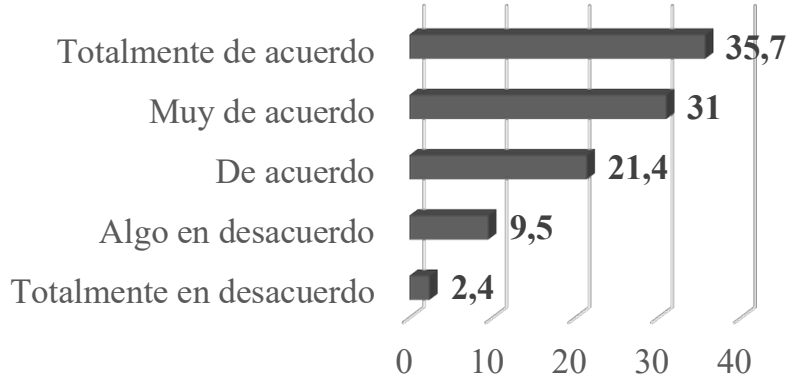

Figura 2: Me ha parecido provechoso trabajar contenidos de la asignatura de 'Mitología Clásica y Cultura Europea' en inglés. 
Además, la eficiencia de la actividad interdisciplinar también viene avalada por la propuesta metodológica a través de la cual el alumnado prepara una presentación oral en EALM que repite, mejorada, tras el feeddback del profesorado, en Inglés I (Figura $3)$, por lo que el esfuerzo empleado es menor con respecto a otros cursos en los que las presentaciones orales en las asignaturas de Inglés I y EALM versaban sobre contenidos diferentes, lo que se traduce en un elevado nivel de satisfacción $(73,8 \%)$ :

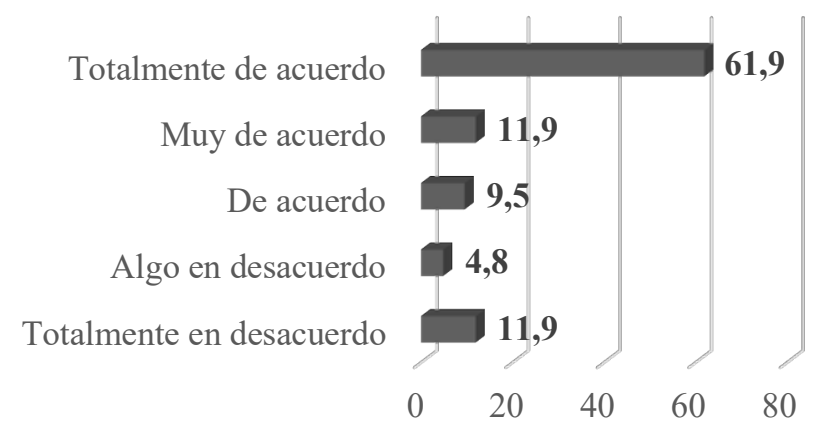

Figura 3: Me ha resultado útil realizar la presentación dos veces para poder mejorarla.

El alumnado también valoró positivamente el hecho de que esta actividad interdisciplinar le hubiese ayudado a consolidar el vocabulario trabajado en Inglés I (31\% Totalmente de acuerdo, 38,1\% Muy de acuerdo). Con respecto a la tarea de trabajo en equipo, si bien desde el punto de vista metodológico no se diseñó la actividad de manera guiada y coordinada, los estudiantes admitieron que la actividad del trabajo en equipo les resultó beneficiosa, a pesar de las dificultades implícitas que pueda llevar consigo (33,3\% Totalmente de acuerdo, 33,3\% Muy de acuerdo, De acuerdo 23,8\%, Algo en desacuerdo 9,5\%). Por último, queremos resaltar que el $64,3 \%$ de los estudiantes prefirió poder elegir el tema de las presentaciones orales a que fuese un tema preestablecido por el equipo docente.

El análisis cualitativo de los 8 comentarios obtenidos en la pregunta abierta de la encuesta (¿Cómo se podría mejorar esta actividad?) nos revela lo siguiente: tres estudiantes declararon que la actividad interdisciplinar entre las tres asignaturas les había parecido "perfecta", "muy entretenida e interesante" y que "no habría nada que mejorar". Los otros cinco comentarios ofrecen sugerencias sobre cuestiones organizativas y para la mejora del diseño de las actividades: asegurar que todo el alumnado esté asignado a un grupo, dar más tiempo para la preparación de las presentaciones orales, aumentar el límite de tiempo de 5-7 minutos por grupo para las presentaciones orales, y ofrecer una variedad mayor de temas en las presentaciones orales para, de este modo, poder "afianzar muchos más términos y vocabulario".

Como conclusión, el impacto de la actividad interdisciplinar llevada a cabo ha sido positiva para el alumnado tal y como se desprende de los resultados de la encuesta de satisfacción. Parece que esta agrupación e integración de contenidos ha fomentado su motivación y les ha permitido observar cómo la coordinación entre contenidos diversos es posible y experimentar cómo esta metodología conduce a un aprendizaje más comprensivo. Diversos estudios han resaltado los beneficios que ofrece la interdisciplinaridad y el impacto de las actividades interdisciplinares en el nivel universitario
(Ackerman, 1988; Carvajal Escobar, 2010; Field, 1994; Fraile Aranda, 2012; Pozuelos Estrada, Rodríguez Mirana y Travé González, 2012; Quintá, Maass, Orta, Trigos y Sabulsky, 2014) y nuestra propuesta pretende ser una evidencia más del carácter integrador, motivador $\mathrm{y}$ facilitador de este tipo de tareas interdisciplinares.

\section{CONCLUSIONES}

Nuestra meta ha sido impregnar de un espíritu globalizador e integrador las enseñanzas del Grado en Lenguas Modernas a partir de una experiencia que ha permitido al alumnado aplicar e interrelacionar los conocimientos adquiridos en tres asignaturas. La experiencia presentada confirma la conveniencia de una coordinación interdisciplinar, que se ha modulado, como se ha demostrado, de forma eficiente: se han aunado contenidos, metodología y compartido sistemas de evaluación, y todo ello a partir de un producto cultural del siglo XXI que ha servido de hilo conductor. Sin duda, la temática de la película elegida reforzó las bondades del enfoque interdisciplinar, en cuanto al fomento de la reflexión crítica y la conexión de la universidad con la realidad que la circunda. Es importante señalar que la obra es susceptible de ser estudiada desde diferentes perspectivas adaptándose así a la diversidad de alumnado existente en el aula: desde una perspectiva de género (tal como ya se hizo en MCCE en el curso 2019-20) o desde el ámbito de la Psicología (son varios los estudios que abordan la película desde este prisma, entre otros Bazzoli (2009)).

De otro lado, la innovación no constituye un cambio puntual $\mathrm{y}$ aislado, sino que debe entenderse como un proceso, un conjunto de acciones sostenidas en el tiempo. Conviene apuntar en ese sentido que esta experiencia tiene su origen en una práctica tímidamente desarrollada en el curso anterior (20192020), que vinculaba dos de las tres asignaturas que ahora son protagonistas (Inglés I y MCCE). En búsqueda de la mejora y afianzamiento de aquella se aunó (en el curso 2020-2021) otra asignatura (EALM) que, sirviendo de amalgama, reforzara el efecto sinérgico. Así configurada esta práctica interdisciplinar, consideramos que puede seguir aplicándose de forma habitual, y, es más, dados los resultados alcanzados, es nuestra intención proseguir desarrollándola en los cursos venideros en aras de su consolidación. Pretendemos, además, centrar nuestros esfuerzos en contribuir al desarrollo de las capacidades de nuestro alumnado para el trabajo en equipo, competencia cada vez más demandada en nuestra sociedad actual, y que está asociado a dos de las actividades de esta experiencia interdisciplinar: la elaboración de presentaciones orales en grupo. Para ello, adaptaremos la metodología de trabajo en equipo propuesta por Lerís, Fidalgo Blanco y Sein-Echaluce Lacleta (2014), Comprehensive Training Model of the Teamwork Competence, que ofrecerá al alumnado formación y entrenamiento sobre el trabajo en equipo y permitirá al profesorado ver la evolución del trabajo individual y de equipo desarrollados, facilitando su evaluación a lo largo de las distintas etapas del proceso del trabajo en equipo.

Por último, pese a que la transferibilidad constituye un rasgo que no se cumple tan fácilmente en la innovación educativa aplicada en el aula (Sein-Echaluce, Fidalgo Blanco, y GarcíaPeñalvo, 2019, p. 618), consideramos que la práctica que aquí se recoge es transferible a otros contextos. Percibimos su 
viabilidad en virtud de posibles relaciones entre otras asignaturas de los módulos antes referidos: sirvan de ejemplos cercanos las asignaturas también de primer curso Inglés II (Módulo I. La Lengua inglesa: aspectos teóricos y prácticos módulo de Lengua), EALM (Módulo VI. Asignaturas instrumentales, metodológicas, de orientación al desempeño profesional y de formación en valores) y Tradición literaria y cultura popular (Módulo IV. Literatura), al ser susceptibles de interrelacionarse, como en la experiencia que aquí presentamos, a partir de un mismo producto cultural -de la alta cultura o de la cultura de masas- estudiado en virtud de los fenómenos de tradición y recepción clásicas. Y es más, podría ampliarse esta red de relaciones a partir de la lengua minor del Grado en Lenguas Modernas, Francés I y Francés II (Módulo II. Segunda lengua extranjera), impartidas también en primer curso. Así el alumnado desde el inicio del Grado estaría familiarizándose con este enfoque interdisciplinar, pudiendo desde ese momento percibir con normalidad la interrelación de conocimientos, y, si estas prácticas se ampliaran a cursos superiores, sin duda quedarían bien asentadas y el alumnado estaría más preparado para enfrentase a la resolución de los complejos problemas que la realidad plantea, para la que se requiere una visión holística.

\section{REFERENCIAS}

Ackerman, P. (1998). Determinants of individual differences during skill acquisition: Cognitive abilities and information processing. Journal of Experimental Psychology, 117(3), 288-318.

Balló J. y Pérez X. (2010). La semilla inmortal: los argumentos universales en el cine. Barcelona, Anagrama.

Bazzoli, M. (2009). The metamorphoses of the Pygmalion Myth: A narrative critique of Lars and the Real Girl, Conference Papers January 2009 National Communication Association.

Brown, S. K. (2010). Popular films in the EFL classroom: Study of methodology. Procedia Social and Behavioral Sciences, 3, 45-54.

Carvajal Escobar, Y. (2010). Interdisciplinariedad: desafío para la educación superior y la investigación. Luna Azul, 31, 156-169.

Consejo de Europa (2002). Marco común europeo de referencia para las lenguas: aprendizaje, enseñanza, evaluación. Madrid: Anaya e Instituto Cervantes.

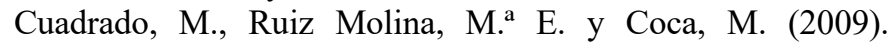
Participación y rendimiento del estudiante universitario en un proyecto docente interdisciplinar, bilingüe y virtual. Revista de Educación, 348, 505-518.

Fidalgo Blanco, A., Sein-Echaluce Lacleta, M. ${ }^{a}$ L., GarcíaPeñalvo, F. J. y Balbín Bastidas, A. Ma . (2019). Método para diseñar buenas prácticas de innovación educativa docente: percepción del profesorado. $V$ Congreso
Internacional sobre Aprendizaje, Innovación y Competitividad (CINAIC 2019). DOI: 10.26754/CINAIC.2019.0127

Field, M. (1994). Assessing interdisciplinary learning. New Directions for Teaching and Learning, 58, 69-84.

Fraile Aranda, A. (2012). Evaluación formativa e interdisciplinariedad: Análisis de dos asignaturas con el mismo sistema de evaluación. Psychology, Society, \& Education, 4(1), 5-16.

Fundación Española para la Ciencia y la Tecnología. (2007). Libro Blanco en Investigación en Humanidades. ISBN/NIPO: 84-690-2438-8.

Goctu, R. (2017). Using movies in the EFL classrooms. European Journal of Language and Literature Studies, 3(2), 121-124.

Ismaili, M. (2013). The Effectiveness of Using Movies in the EFL Classroom - A Study Conducted at South East European University. Academic Journal of Interdisciplinary Studies, 2(4), 121-132.

Lerís, D., Fidalgo Blanco, Á., Sein-Echaluce Lacleta y $\mathrm{M}^{\mathrm{a}} \mathrm{L}$. (2014). A comprehensive training model of the teamwork competence. International Journal of Learning and Intellectual Capital, 11(1), 1-19.

Lin, H. (2008). Opportunities and challenges for interdisciplinary research and education. Journal of Natural Resources and Life Sciences Education, 37, 8391.

López-Roldán, P. y Fachelli, S. (2015). Metodología de la investigación social cuantitativa. Universidad Autónoma de Barcelona.

Oxford, R. (1999). Language learning strategies: What every teacher should know. Boston, MA: Heinle \& Heinle.

Pozuelos Estrada, F. J., Rodríguez Miranda, F. y Travé González, G. (2012). El enfoque interdisciplinar en la enseñanza universitaria y el aprendizaje basado en la investigación. Un estudio de caso en el marco de la formación. Revista de Educación, 357, 561-585.

Quintá, M. C. (Coord.), Maass, M., Orta, M., Trigos, L. y Sabulsky, G. (2014). Estrategias para la formación interdisciplinar en las áreas de Humanidades y Ciencias Sociales y en Artes, Arquitectura y Diseño. Estrategias Docentes para la Formación Interdisciplinar en Educación Superior. Red Innova CESAL, 99-124.

Seferoglu, G. (2008). Using feature films in language classes. Education Studies, 34(1), 1-9.

Sein-Echaluce, M.L., Fidalgo Blanco, A., y García-Peñalvo. F.J. (2019). Diseño de un proyecto de innovación educativa docente a partir de indicadores transferibles entre distintos contextos. V Congreso Internacional sobre Aprendizaje, Innovación y Competitividad (CINAIC 2019). DOI:10.26754/CINAIC.2019.0126.

Wall, S. L. y Shankar, I. (2008). Adventures in transdisciplinary learning. Studies in Higher Education, 33(5), 551-565. 